\title{
Are Vicinal Metal Surfaces Stable?
}

\author{
Frenken, J. W. M.; Stoltze, Per
}

Published in:

Physical Review Letters

Link to article, DOI:

10.1103/PhysRevLett.82.3500

Publication date:

1999

\section{Document Version}

Publisher's PDF, also known as Version of record

Link back to DTU Orbit

\section{Citation (APA):}

Frenken, J. W. M., \& Stoltze, P. (1999). Are Vicinal Metal Surfaces Stable? Physical Review Letters, 82(17), 3500-3503. https://doi.org/10.1103/PhysRevLett.82.3500

\section{General rights}

Copyright and moral rights for the publications made accessible in the public portal are retained by the authors and/or other copyright owners and it is a condition of accessing publications that users recognise and abide by the legal requirements associated with these rights.

- Users may download and print one copy of any publication from the public portal for the purpose of private study or research.

- You may not further distribute the material or use it for any profit-making activity or commercial gain

- You may freely distribute the URL identifying the publication in the public portal

If you believe that this document breaches copyright please contact us providing details, and we will remove access to the work immediately and investigate your claim. 


\title{
Are Vicinal Metal Surfaces Stable?
}

\author{
J. W. M. Frenken \\ Kamerlingh Onnes Laboratory, Leiden University, P.O. Box 9504, 2300 RA Leiden, The Netherlands \\ P. Stoltze \\ Center for Atomic-scale Materials Physics, Department of Physics, Technical University of Denmark, \\ DK-2800 Lyngby, Denmark \\ (Received 13 November 1998)
}

\begin{abstract}
We use effective medium theory to demonstrate that the energies of many metal surfaces are lowered when these surfaces are replaced by facets with lower-index orientations. This implies that the lowtemperature equilibrium shapes of many metal crystals should be heavily faceted. The predicted instability of vicinal metal surfaces is at variance with the almost generally observed stability of these surfaces. We argue that the unstable orientations undergo a defaceting transition at relatively low temperatures, driven by the high vibrational entropy of steps. [S0031-9007(99)09027-4]
\end{abstract}

PACS numbers: 68.35.Md, 65.50.+m, 68.35.Ja, 68.35.Rh

The interactions between neighboring steps on vicinal surfaces play a crucial role in the surface morphology and the crystal equilibrium shape [1-4]. It is generally assumed that steps repel each other, and that the distance dependence of this repulsion ensures the stability of the surface. Here, we predict that the distance dependence of the step interaction energy on many metals is such that appreciable ranges of orientations are, in fact, unstable. We claim that the observed stability of these surfaces results entirely from the entropic contribution of step vibrations to the surface free energy. We illustrate this idea with effective medium theory (EMT) calculations [5] for several fcc metals. With our claim, we put the free energy balance on the equilibrium contour of metal crystals in a completely new perspective.

The free energy $f(\theta)$ of a vicinal surface, misoriented with respect to a low-index orientation by an angle $\theta$, is usually expanded as follows:

$$
f(\theta)=f(0)+\frac{b(d)}{d}=f(0)+b(d) \frac{|\tan \theta|}{h},
$$

with

$$
b(d)=\beta+g(d) .
$$

The energy is expressed per unit area of the low-index orientation. $f(0)$ is the free energy of the low-index plane itself. $b(d)$ is the free energy per unit length of a step on the vicinal surface. Its main contribution $\beta$ is the free energy per unit length of an isolated step on the low-index plane. The step interaction free energy per unit length $g$ depends on the average distance $d=h /|\tan \theta|$ between nearest-neighbor steps, where $h$ is the step height. For sufficiently large step distances $d$, the displacement fields around the steps interact with each other as elastic dipole lines, which makes $g(d)$ decay as $d^{-2}[1,6]$. Experiments with crystallization waves on ${ }^{4} \mathrm{He}$ crystals confirm this prediction [7], and various microscopic studies of step distance distributions on semiconductor and metal surfaces are consistent with this simple distance dependence [8-10].
Recently, surface energies and step interaction energies of vicinal metal surfaces at zero temperature have been computed [11-15] with both pairwise and nonpairwise potentials, such as the embedded atom model (EAM). The results of Refs. [11,13] show that at short distances the steps no longer obey the simple $d^{-2}$ repulsion law, and an additional $d^{-3}$ attraction seems to be present [11]. For very small step separations, the short-range attraction leads to an inflection in the dependence of $g$ on $d$, which, as we demonstrate below, results in the instability of a range of vicinal orientations.

We have computed the zero-temperature energies $F(\theta)$ [16] for large numbers of low-index and vicinal surface orientations of $\mathrm{Ag}, \mathrm{Au}, \mathrm{Cu}, \mathrm{Ni}, \mathrm{Pd}$, and $\mathrm{Pt}$, using the EMT potential. We selected as many as 135 nonequivalent orientations distributed over the stereographic triangle, spanned by (100), (110), and (111), together representative for the full crystal shape. Our calculations were performed for a slab geometry with a large unit cell in the form of a parallelepiped. For each surface orientation, the shape and dimensions of the cell were chosen such that, in combination with periodic boundary conditions parallel to the surface, the cell generated the full surface structure, including that of the regularly spaced steps. The cells contained two free surfaces and the lattice constants parallel to the surface were fixed to those corresponding to the bulk equilibrium lattice structure. The calculations were performed for cell thicknesses ranging from 6 to 16 atomic layers, and it was checked that the employed thickness was always large enough that it did not affect the calculated surface energy within our precision of $\pm 1 \times 10^{-5} \mathrm{eV} / \AA^{2}$. Starting from atomic positions on a truncated bulk lattice, we minimized the energy by relaxing the structure according to a combination of steepest descent, Monte Carlo and molecular dynamics methods.

Figure 1a shows the calculated energies $F(\theta)$ versus $\tan (\theta)$, for an angular range of $54.74^{\circ}$ from $\operatorname{Ag}(100)$ to $\mathrm{Ag}(111)$. If the step interactions $G$ [16] were absent, the 

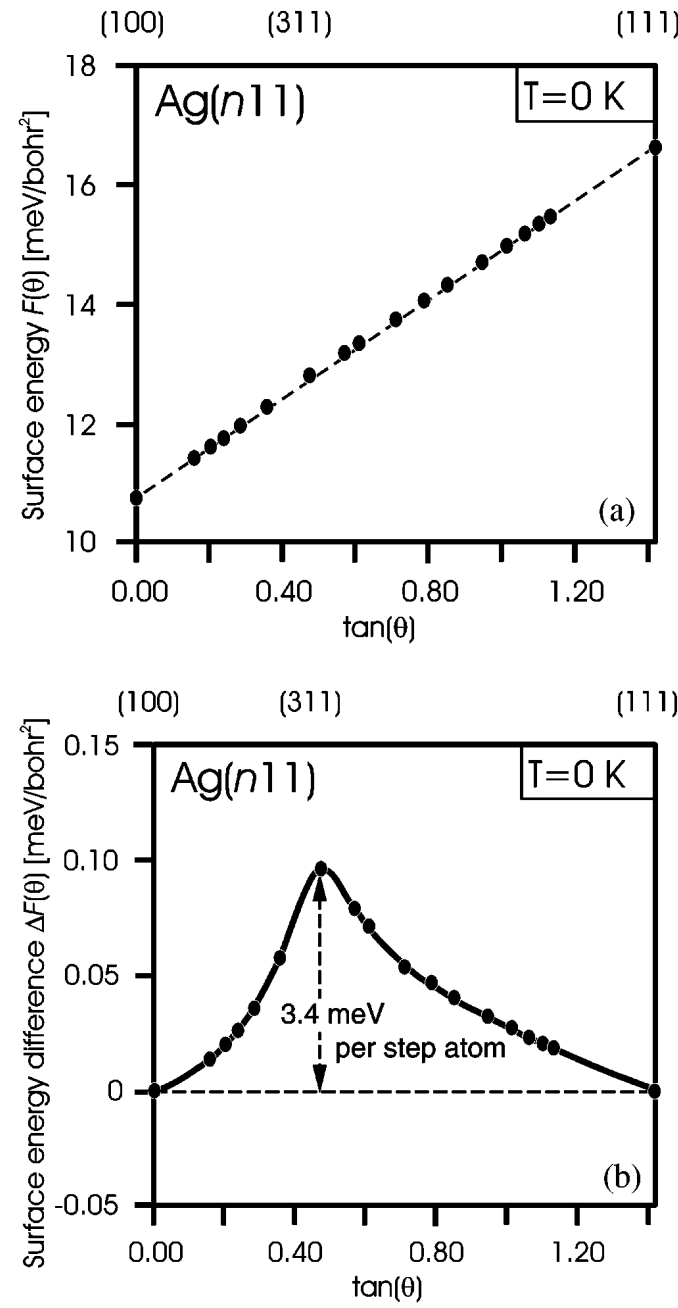

FIG. 1. (a) Calculated energies of $\operatorname{Ag}(n 11)$ surfaces at $0 \mathrm{~K}$. $F$ is expressed per unit of area projected on the (100) surface, and is plotted against the tangent of the angle $\theta$ with (100). The dashed straight line connects the (100) and (111) orientations and demonstrates that all intermediate orientations lower their energy when broken up into (100) and (111) facets. (b) Difference between the surface energy $F(\theta)$ at $0 \mathrm{~K}$ and the corresponding linear combination of energies for $\operatorname{Ag}(100)$ and $\operatorname{Ag}(111)$ facets [dashed line in panel (a)]

energies would fall on a straight line [see Eq. (1)]. Although the energies almost lie on the dashed straight line between (100) and (111), there is a small, yet significant deviation: all intermediate orientations have energies slightly above the line. The difference $\Delta F(\theta)$ between the energies $F(\theta)$ and the dashed line in Fig. 1a is plotted in Fig. 1b. The fact that part of this curve is concave (negative curvature) has the consequence that a range of orientations is unstable [3]. The unstable surfaces are lowered in energy when replaced by a linear combination of other orientations. In Fig. 1 the surface energy of such a linear combination can simply be determined by linear interpolation; each orientation lowers its energy to the value on the dashed straight line, by rearranging into (100) and (111) facets. We see that the modest nonconvexity has a dramatic consequence: the entire range of orientations between (100) and (111) is unstable.

In general, for concave surface (free) energy curves, the lowest-energy tie line identifies the most favorable facet combinations [3]. In the past, several examples have been found experimentally, where the surface free energy curve contains a concave region as a result of the existence of two possible "states" for the surface, e.g., reconstructed vs nonreconstructed, and surface-melted vs dry [17-19]. In those cases the nonconvexity arises when the free energy curves for these two states cross because the state with the higher surface free energy $f(0)$ at $\theta=0$ has the lower step free energy $\beta$. Here, we find nonconvexity within one state, directly coming from the distance dependence of the step interaction energy.

Our calculations show that at zero temperature almost all orientations $\mathrm{Ag}(\mathrm{hkl})$ facet into linear combinations of (111), (100), and an orientation close to (110). The familiar Wulff construction allows one to obtain the equilibrium crystal shape from the surface (free) energies [3,4]. The instability of the high-index Ag surfaces with respect to faceting into the three low-index orientations corresponds to the complete absence of almost all orientations from the zero-temperature crystal shape. Figure 2 shows the equilibrium shape of an $\mathrm{Ag}$ crystal calculated from the 135 calculated surface energies. It consists almost entirely of flat $\{100\},\{110\}$, and $\{111\}$ facets, connected with each other by sharp edges. There is a modest, partial rounding of the edges between the $\{110\}$, and $\{111\}$ facets and of all corners, where a limited set of high-index orientations is marginally stable.

We have obtained results for $\mathrm{Cu}, \mathrm{Ni}, \mathrm{Pd}, \mathrm{Pt}$, and $\mathrm{Au}$ surfaces, that are qualitatively similar to those for $\mathrm{Ag}$, although the ranges of unstable orientations are different for each material.

One may wonder whether the computed instabilities simply reflect an imperfection of the employed model

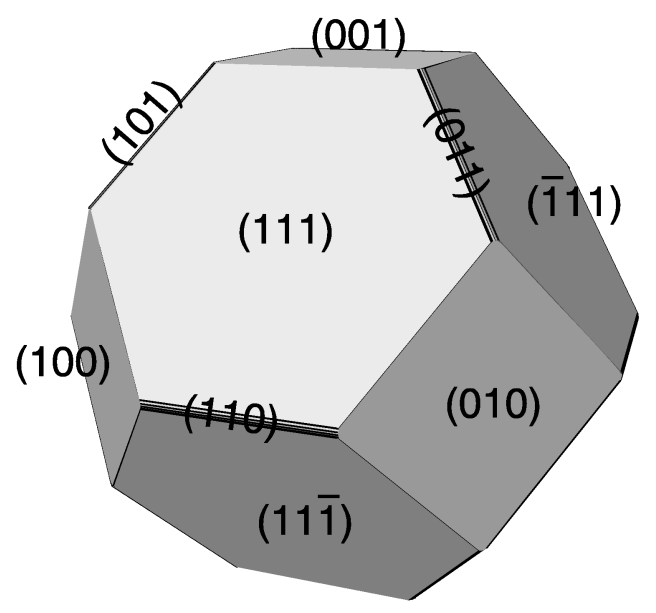

FIG. 2. The equilibrium shape of an $\mathrm{Ag}$ single crystal at zero temperature, obtained via the Wulff construction from the computed surface energies. 
for the metallic interactions. The fact that the surface energies computed for Ag with the EAM potential [11] are almost identical to our present EMT results for $\mathrm{Ag}$, and the observation that the instabilities are found for several materials, demonstrate that these instabilities should at least be viewed as characteristic for EMT-type potentials. The energy difference between an unstable vicinal surface and the lower-energy linear combination of (111), (100), and (110) facets typically amounts to $1 \%$. The EMT surely does not allow us to calculate surface energies with an absolute accuracy anywhere near $1 \%$. Nevertheless, here we concentrate on surface energy ratios between different orientations of the same material, and these ratios are much more accurate than the energies themselves. The interactions between distant steps are indirect and do not depend on the atomic-scale details of the potential, as long as the resulting elastic constants are properly predicted or fitted by the potential, which is the case for both the EMT and the EAM.

Assuming that our prediction of the zero-temperature instability of many vicinal orientations is correct, we need to explain the widely observed stability of these surfaces against faceting $[9,10,20,21]$. We propose that the vicinal surfaces are almost always stabilized by entropy. There are two sources of entropy that may change the difference in free energy between a vicinal surface and a combination of low-index facets. Above the roughening temperature $T_{R}$, the steps meander over the vicinal surface. The entropy gain involved in the meandering is high enough to make the free energy cost of height fluctuations on the vicinal surface vanish at $T_{R}$ [21]. However, the meandering entropy cannot explain the stability of vicinal surfaces well below $T_{R}$. From a typical value of $0.12 \mathrm{eV}$ for the kink formation energy on stepped $\mathrm{Ag}$ and $\mathrm{Cu}$ surfaces [10,20,22], we calculate an upper estimate for the free energy lowering at $300 \mathrm{~K}$ due to the meandering of merely $0.6 \mathrm{meV} / \mathrm{step}$ atom. At $100 \mathrm{~K}$ this has even reduced to $20 \mathrm{neV} / \mathrm{step}$ atom.

Thermal vibrations form the second source of entropy. The reduced coordination of the step atoms on a vicinal surface makes the average vibration amplitude of vicinal surface atoms significantly higher than the vibration amplitude on low-index surfaces [23-25]. As a consequence, the excess vibrational entropy of a vicinal surface is higher than that of the corresponding low-index facet combination. For a proper calculation of the differences between the excess entropies, the full vibrational energy spectrum has to be computed for each of the surface orientations involved [23,24]. Here, we give only a simple estimate of the effect for a surface with the $(n 11)$ orientation. This surface consists of (100) terraces with a width of $n / 2$ atoms, separated by steps. In the case of faceting, all steps are brought together to form a (111) terrace, and the step-free remainder of the surface has the (100) orientation. We assume for simplicity that the surface atoms are harmonic, isotropically vibrating Einstein oscillators, and that the step atoms on the $(n 11)$ surface have a vi- bration amplitude $\sigma^{\text {step }}$ that is $20 \%$ higher than that on the (111) surface, $\sigma^{(111)}$ [23-25]. The other atoms at the (n11) surface are assumed to have the same vibration amplitude as the terrace atoms on the (100) surface [25]. Under these assumptions, the only difference in vibrational entropy comes from the step atoms that are reduced in vibration amplitude when they are reorganized into a (111) facet. The entropy difference per step atom is

$$
\begin{aligned}
\Delta S & =S^{\text {step }}-S^{(111)}=3 k_{B} \ln \frac{\sigma^{\text {step }}}{\sigma^{(111)}} \simeq 0.6 k_{B} \\
& =0.05(\mathrm{meV} / \mathrm{K}) / \text { step atom },
\end{aligned}
$$

where the factor 3 accounts for the three orthogonal directions of vibration. At room temperature, this entropy difference reduces the free energy per step atom on the (n11) surface by as much as $15 \mathrm{meV}$ with respect to atoms on the (111) facet. This is much more than the free energy reduction needed to stabilize all $(n 11)$ surfaces (see Fig. 1). The situation is similar for other vicinal orientations and other materials. This brings us to the important conclusion that the step vibrational entropy is sufficiently large to account for the stability of the vicinal orientations of most metals at room temperature.

We stay with the $(n 11)$ orientations of Ag to illustrate the temperature dependence of the instability. Above $0 \mathrm{~K}$, these vicinal surfaces continuously lower their free energy with respect to the (111) and (100) orientations. For simplicity, we assume that the ratios between the vibration amplitudes do not depend on temperature, and that at each temperature $T$, the step-free energies for all orientations (n11) are lowered by the same amount, $0.6 k_{B} T$ per step atom. As indicated in Fig. 3, this leads to a reduction of the surface free energy that is strongest for (311). At relatively low temperatures of approximately 35 and $65 \mathrm{~K}$ small ranges of vicinal orientations become stable around (100) and (111), respectively. When the temperature is increased further, these two stable ranges grow, as is illustrated by the double-tangent construction in Fig. 3 . At approximately $150 \mathrm{~K}$, the unstable range is reduced to zero at an orientation close to (311). This combination of temperature and orientation is the critical point $\left(T_{c}, \theta_{c}\right)$ for the faceting transition in this zone of orientations. A similar reduction of the range of unstable orientations with increasing temperature takes place on the other vicinal orientations that are faceted into (111), (110), and (100) at low temperatures. In this way, almost all orientations are stabilized on the crystal equilibrium shape of most metals, at temperatures well below $300 \mathrm{~K}$.

There is a simple reason why the faceting has not been observed in low-temperature studies of vicinal metal surfaces [26,27]. Well-ordered metal surfaces are almost always prepared above room temperature, where the vicinal orientations are stable. When the metal is cooled, the diffusion is quenched well before the flat vicinal surfaces become unstable [28].

Finally, it is possible that the vibrational frequency spectra on the low- and high-index surfaces are 


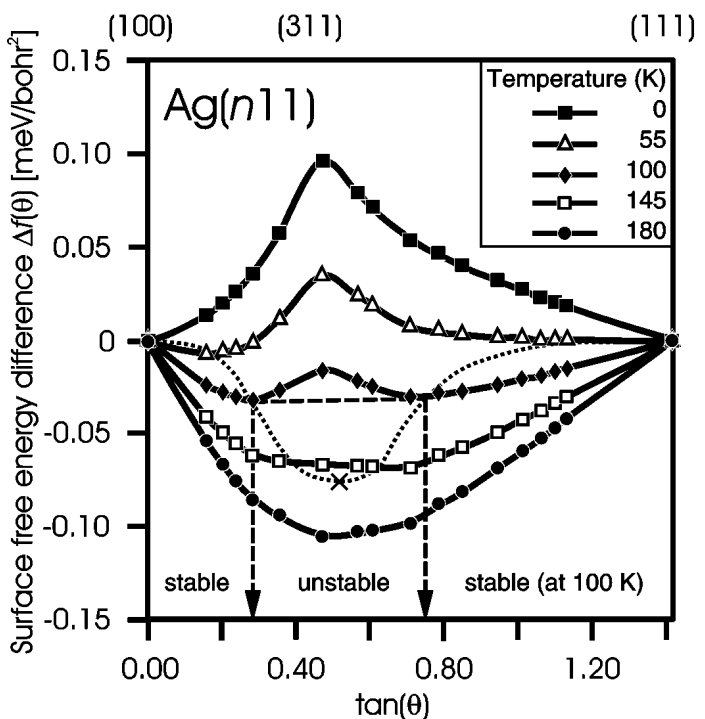

FIG. 3. Temperature dependence of the difference $\Delta f(\theta)$ between the surface free energy and the corresponding linear combination of surface free energies for $\operatorname{Ag}(100)$ and $\operatorname{Ag}(111)$ facets at $0 \mathrm{~K}$ (solid squares), $55 \mathrm{~K}$ (open triangles), $100 \mathrm{~K}$ (solid diamonds), $145 \mathrm{~K}$ (open squares), and $180 \mathrm{~K}$ (solid circles) (see text). The dashed straight line indicates the double-tangent construction at $100 \mathrm{~K}$. The dotted curve connects the tangent points obtained at each temperature, and the cross indicates the critical point $\left(T_{c}, \theta_{c}\right)$ at approximately $155 \mathrm{~K}$ for the faceting in this zone of orientations.

sufficiently different, that the difference in zero-point vibrational energies already stabilizes some vicinal surfaces at $0 \mathrm{~K}$. In particular, the presence of low-frequency step modes will have a direct influence on the low-temperature surface energy balance. We estimate that this will affect only a few vicinal orientations, and that for most vicinal surfaces the faceting involves energy differences that are at least an order of magnitude larger than the zero-point vibration energy difference.

In summary, total energy calculations of several fcc metal surfaces suggest that most surface orientations are unstable at $0 \mathrm{~K}$, and the true equilibrium shape should be formed predominantly by $\{111\},\{100\}$, and $\{110\}$ facets. The difference in vibrational characteristics between step atoms and atoms on close-packed terraces leads to an excess vibrational entropy on the vicinal surfaces, that we estimate to be sufficiently large to make most orientations of most materials stable at room temperature.

We are grateful to E. Tosatti, J. Wettlaufer, T. Rahman, J. K. Nørskov, and D. Frenkel for valuable discussions. This work is part of the research program of the "Stichting voor Fundamenteel Onderzoek der Materie (FOM)," which is financially supported by the "Nederlandse organisatie voor Wetenschappelijke Onderzoek (NWO)." The Center for Atomic-scale Materials Physics is sponsored by the Danish National Research Foundation.
[1] E. D. Williams, Surf. Sci. 299/300, 502 (1994).

[2] C. Jayakaprash, C. Rottman, and W. F. Saam, Phys. Rev. B 30, 6549 (1984).

[3] C. Herring, in Structure and Properties of Solid Surfaces, edited by R. Gomer and C.S. Smith (University of Chicago Press, Chicago, 1953), p. 5.

[4] G. Wulff, Z. Kristall. Mineral. 34, 449 (1901).

[5] K. W. Jacobsen, J. K. Nørskov, and P. Stoltze, Surf. Sci. 366, 394 (1996).

[6] V. I. Marchenko and A. Ya. Parshin, Sov. Phys. JETP 52, 129 (1980).

[7] E. Rolley et al., Phys. Rev. Lett. 72, 872 (1994).

[8] C. Alfonso et al., Surf. Sci. 262, 371 (1992).

[9] M. Giesen, Surf. Sci. 370, 55 (1997).

[10] L. Barbier et al., Surf. Sci. 345, 197 (1996).

[11] R. Najafabadi and D. J. Srolovitz, Surf. Sci. 317, 221 (1994).

[12] L. E. Shilkrot and D. J. Srolovitz, Phys. Rev. B 53, 11120 (1996).

[13] D. Wolf and J. A. Jaszczak, Surf. Sci. 277, 301 (1992).

[14] R. C. Nelson et al., Surf. Sci. 295, 462 (1993).

[15] K. D. Hammonds and R. M. Lynden-Bell, Surf. Sci. 278, 437 (1992).

[16] We denote free energies by lowercase symbols and zerotemperature energies by capital symbols.

[17] P. Nozières, in Solids Far from Equilibrium, edited by C. Godrèche (Cambridge University Press, Cambridge, 1992), p. 1.

[18] J.W. M. Frenken and H. M. van Pinxteren, in The Chemical Physics of Solid Surfaces: Phase Transitions and Adsorbate Restructuring at Metal Surfaces, edited by D. A. King and D.P. Woodruff (Elsevier, Amsterdam, 1994), Vol. 7, p. 259.

[19] E. D. Williams et al., Surf. Sci. 294, 219 (1993); 310, 451 (1994).

[20] M. S. Hoogeman et al., Phys. Rev. B 53, R13 299 (1996).

[21] J. Lapujoulade, Surf. Sci. Rep. 20, 191 (1994), and references therein.

[22] M. Giesen-Seibert et al., Surf. Sci. 329, 47 (1995).

[23] S. Durukanoğlu, A. Kara, and T. S. Rahman, Phys. Rev. B 55, 13894 (1997).

[24] A. Kara, S. Durukanoğlu, and T.S. Rahman, J. Chem. Phys. 106, 2031 (1997).

[25] Z.-J. Tian and J.E. Black, Surf. Sci. 303, 395 (1994).

[26] Surface reconstruction on low-index orientations can lead to faceting of vicinal surfaces into so-called "magic" orientations: A. Bartolini, F. Ercolessi, and E. Tosatti, Phys. Rev. Lett. 63, 872 (1989). This has been observed on vicinal gold surfaces, but falls outside the scope of the present article: J.C. Heyraud and J. J. Métois, Acta Metall. 28, 1789 (1980); J. Cryst. Growth 50, 571 (1980); Y. Samson et al., Surf. Sci. 315, L969 (1994).

[27] The reversible high-temperature faceting that has been observed in x-ray diffraction experiments on vicinal $\mathrm{Ag}$ and $\mathrm{Cu}$ surfaces seems to be of a different nature than the faceting transition predicted here: B.M. Ocko and S. G. J. Mochrie, Phys. Rev. B 38, 7378 (1988); I. K. Robinson et al., Phys. Rev. Lett. 67, 1890 (1991).

[28] F. Fabre et al., Surf. Sci. 175, L693 (1986). 